\title{
BMJ Open Retrospective qualitative study evaluating the application of IG4 curriculum: an adaptable concept for holistic surgical education
}

\author{
lakovos Theodoulou, ${ }^{1}$ Michail Sideris (D) , ${ }^{2}$ Kola Lawal, ${ }^{1}$ Marios Nicolaides, ${ }^{3}$ \\ Aikaterini Dedeilia, ${ }^{4}$ Elif Iliria Emin, ${ }^{1}$ Georgios Tsoulfas, ${ }^{5}$ Vassilios Papalois, ${ }^{6,7}$ \\ George Velmahos, ${ }^{8}$ Apostolos Papalois ${ }^{9,10}$
}

To cite: Theodoulou I, Sideris M, Lawal K, et al. Retrospective qualitative study evaluating the application of IG4 curriculum: an adaptable concept for holistic surgical education. BMJ Open 2020;10:e033181. doi:10.1136/ bmjopen-2019-033181

- Prepublication history for this paper is available online. To view these files, please visit the journal online ().

IT and MS contributed equally.

IT and MS are joint first authors.

Received 25 July 2019

Revised 16 January 2020

Accepted 21 January 2020

Check for updates

(C) Author(s) (or their employer(s)) 2020. Re-use permitted under CC BY-NC. No commercial re-use. See rights and permissions. Published by BMJ.

For numbered affiliations see end of article.

Correspondence to

Dr Michail Sideris;

msideris@nhs.net

\section{ABSTRACT}

Objectives Faced with a costly and demanding learning curve of surgical skills acquisition, the growing necessity for improved surgical curricula has now become irrefutable. We took this opportunity to formulate a teaching framework with the capacity to provide holistic surgical education at the undergraduate level.

Setting Data collection was conducted in all the relevant healthcare centres the participants worked in. Where this was not possible, interviews were held in quiet public places.

Participants We performed an in-depth retrospective evaluation of a proposed curriculum, through semistructured interviews with 10 participants. A targeted sampling technique was employed in order to identify senior academics with specialist knowledge in surgical education. Recruitment was ceased on reaching data saturation after which thematic data analysis was performed using NVivo 11.

Results Thematic analysis yielded a total of 4 main themes and 29 daughter nodes. Majority of study participants agreed that the current landscape of basic surgical education is deficient at multiple levels. While simulation cannot replace surgical skills acquisition taking place in operating rooms, it can be catalytic in the transition of students to postgraduate training. Our study concluded that a standardised format of surgical teaching is essential, and that the Integrated Generation 4 (IG4) framework provides an excellent starting point.

Conclusions Through expert opinion, IG4 has been validated for its capacity to effectively accommodate learning in a safer and more efficacious environment. Moreover, we support that through dissemination of IG4, we can instil a sense of motivation to students as well as develop robust data sets, which will be amenable to data analysis through the application of more sophisticated methodologies.

\section{INTRODUCTION}

Following the global recession in 2008, healthcare systems around the world have struggled to meet desirable standards of care, due to significant diversion of funds away from health and social care. Education has
Strengths and limitations of this study

- A comprehensive in-depth qualitative evaluation of a simulation-based surgical curriculum.

- Triangulation was achieved by conducting semistructured interviews with surgeons across two continents.

- The proposed curriculum design provides a reproducible and adaptable curriculum for use in many educational settings.

- Future work should focus on supplementing this study with collection of quantitative data.

unequivocally suffered in similar patterns, which has rendered the delivery of high quality and cost-effective surgical training increasingly challenging. In view of these developments, simulation-based learning (SBL) has evolved to take up a major role in global efforts and to address the costly and demanding learning curve of surgical skill acquisition. ${ }^{1}$ SBL has been proven to improve the safety and efficacy of in-hospital training, as well as to reconcile a frequently occurring 'conflict' between training time requirements and safe care provision. ${ }^{2}$

The necessity to re-evaluate the effectiveness of current curricula is not solely based on increasing financial and time pressures, but also derives from poor satisfaction levels with surgical teaching as reported by medical students. ${ }^{3}$ Overall, this points to a systemic problem which contributes to newly qualified doctors feeling less prepared for jobs in surgical specialities when compared with medical specialities. ${ }^{4}$ Furthermore, the Royal College of Surgeons (RCS) has recently published 'Future of Surgery', a report setting out a vision in view of the latest challenges for surgical training in the UK. ${ }^{5}$ It outlines the increasing importance of leadership, 
managerial and entrepreneurial skills alongside traits of flexibility and versatility. While surgical education does occupy an important part of medical school, concerns have previously been voiced regarding the limited exposure of students to some surgical specialities as well as the inadequacy of surgical training altogether. ${ }^{6}$ With this shifting landscape, it is unsurprising to see rising attrition rates across various postgraduate surgical pathways. ${ }^{7}$

Faced with this mounting pressure for a change, there is now a strong case for improved and innovative surgical training curricula, which not only reduce attrition rates but also address existing inefficiencies. Considering the multifaceted nature of this problem, a qualitative exploration of the subject was deemed essential, however, recent systematic reviews have failed to identify studies qualitatively appraising the status quo. ${ }^{8}$ We took this opportunity to formulate and test a novel curriculum framework with the capacity to provide holistic surgical education; one which provides participants with a broad overall view of the variety of surgical specialities, through hands-on simulation. The curriculum version described in this study, was developed as part of the research activity related to the 'Essential Management of Surgical Cases' (ESMSC marathon course) - an international simulationbased initiative. The curriculum of ESMSC has undergone continuous improvement since its inception and its most recent published form (Cores Integrated for Research; Ci4R $)^{9}$ combines small group teaching with mixed fidelity in vivo, ex vivo and dry lab simulation. Modules are currently split into four core disciplines: technical skills, non-technical skills, basic science and applied clinical science. The foundations of Ci4R have been described in detail by Sideris $e t a l .^{9} 10$

\section{Aim}

The four main focus areas of this study included: first, to investigate the current state of undergraduate surgical training, based on expert opinions of experienced surgeons; second, to identify challenges in improving current curricula; third, to determine the role of simulation-based learning and lastly, to appraise the Integrated Generation 4 (IG4) framework and the extent to which it addresses identified issues. Finally, we evaluated IG4 against potential adaptability and reproducibility at an international level.

\section{The novel 'Integrated Generation 4' curriculum}

In this study, we present the newest version of the Ci4R: a three-dimensional design titled the Integrated Generation 4 model. IG4 is built on a refined framework which can be adapted based on regional targets and which accommodates the needs of specific surgical specialities (figure 1). IG4 has a three-dimensional design where in addition to ensuring a holistic surgical education by encapsulating skills (x-axis) and knowledge (y-axis), it also positions teaching modules on a spectrum of 'affinity' (z-axis). Affinity can be defined as the degree of generalisability of a module by answering a simple

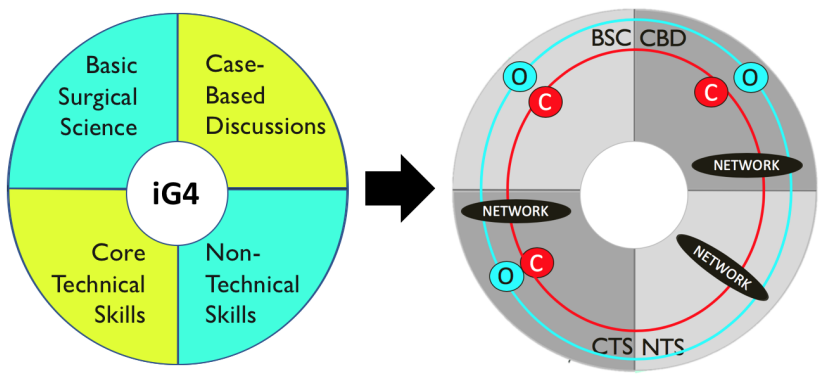

Figure 1 On the left are the four cores of IG4; on the right is a theoretical application of IG4 in orthopaedics $(\mathrm{O})$ and cardiothoracics (C). Network nodes (black) are shareable, core-specific modules across specialities. Different coloured lines connect modules from all four cores that are specific to a single speciality. IG4,Integrated Generation 4

question: how many specialities would benefit from the delivery of this module? The higher the affinity of a new module, the less applicable this module would be to other specialities (eg, intracranial bolt insertion). Considering that our proposed educational modules lie between different surgical specialities, the higher the affinity of one module to a speciality, this automatically renders this module less applicable to other specialities. We invite the reader to think of surgical specialities as 'competing magnets' for the same module. The less attractable a module is to a single speciality, the less its affinity with it, therefore making that module more 'general' and therefore, applicable to more specialities. With the introduction of 'affinity', speciality-specific training can emerge to address the needs of all specialities more holistically. This paves the way for 'Speciality clusters': sets of modules mapped across the four cores, which are designed to meet learning objectives of a single speciality. Meanwhile, affinity characterisation also ensures that two specialities with considerable overlap may continue to share low affinity teaching modules; we coined these as 'network' modules. The positioning of these nodes within the cores is irrelevant; rather, these are shareable modules across specialities. Figure 1, offers a theoretical application of IG4 in two surgical specialities.

\section{Application of IG4}

The IG4 curriculum has been applied across four student cohorts over the last 2 years, each cohort comprising of 40 students from across Europe, the USA and Asia. In addition to having students participating from three different continents, the IG4 has been successfully implemented in two different simulation centres with different infrastructures and simulation capabilities. The important differences of running IG4 across these educational settings were the varying levels of baseline knowledge and skills of students, the often contrasting cultures of learning (eg, paternalistic vs student-driven) and the different simulation capabilities in the simulation centres. Important similarities included the fact that majority of student 
participants were senior medical students (year 3 and above) as well as the fact that the course was delivered by the same faculty with faculty members mainly from the UK, Greece and the USA. Furthermore, IG4 has been unfolded across six different specialities: cardiothoracic surgery, neurosurgery, general surgery, orthopaedic surgery, plastic surgery and interventional radiology. Stemming from the above, one can argue that the IG4 has proven to be an effective teaching framework, across different geographical locations with varying educational settings both in terms of simulation infrastructure and learning cultures.

\section{METHODS}

The explorative nature of the research question required an in-depth evaluation of the views and perspectives of varied group of participants. In an effort to conduct a retrospective evaluation of our proposed curriculum, data collection relied on a qualitative group facilitation technique in a manner similar to the Delphi. ${ }^{11}$ We used semi-structured interviews with 'a panel of informed individuals', ${ }^{12}$ who in this case were carefully handpicked professors of surgery from Europe and the USA. A purposive sampling technique ${ }^{13}$ was employed in order to identify Professors (P1 to P10) with specialist knowledge in surgical education. All experts who were interviewed had previously attended the course as observers and hence, were aware of the previous course curriculum (Ci4R). Recruitment of the study's participants was ceased once data saturation was reached and new themes stopped emerging. Figure 2 provides a diagrammatic representation of the study methodology and table 1 offers a list of participants' characteristics. This study did not involve any patients.

\begin{tabular}{|c|c|c|}
\hline Position & Speciality & Geographical location \\
\hline Professor & Cardiothoracic surgery & Greece \\
\hline Professor & Cardiothoracic surgery & UK \\
\hline Professor & Transplant surgery & UK \\
\hline Professor & Colorectal surgery & UK \\
\hline Professor & Colorectal surgery & UK \\
\hline Professor & Colorectal surgery & USA \\
\hline Professor & Neurosurgery & UK \\
\hline Professor & General surgery & Greece \\
\hline Professor & Trauma surgery & USA \\
\hline Professor & Orthopaedics surgery & Greece \\
\hline
\end{tabular}

Verbal and written informed consents were collected from all participants prior to the study. Interview guides with pre-agreed topics were developed by the course's faculty and formed an aide-memoire for the researchers. The interview process was held in quiet settings to minimise distractions and maintain anonymity, while duration ranged from 30 to $45 \mathrm{~min}$. Parallel note-taking during the interview was performed by a second interviewer to ensure that important trajectories were followed up. Interviews were recorded and transcribed shortly following completion. Concurrent thematic analysis, involved analysing key themes that arrived from each interview, and helped identify and discuss previously unexplored topics in subsequent interviews. ${ }^{14}$

Final data analysis was performed with NVivo 11 (for Mac) which formed a crucial step in the thematic analysis of the completed transcripts. Twenty-nine nodes were initially identified and were further refined in

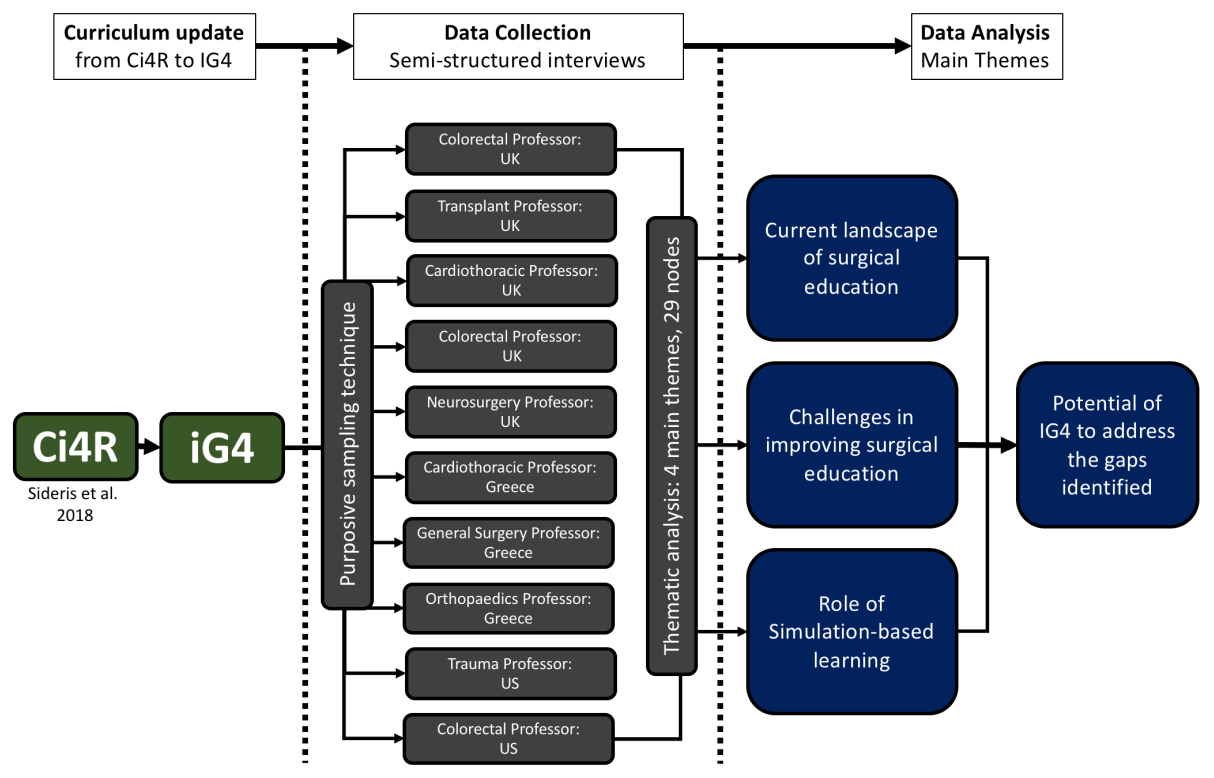

Figure 2 A diagrammatic representation of the adopted methodology. Ci4R,Cores Integrated for Research; IG4, Integrated Generation 4. 
subsequent rounds of data analysis. Analyst triangulation was achieved by engaging all researchers in the identification of codes. ${ }^{1516}$ Several coding rounds were performed, each time focussing on identification of new themes and amalgamation of subsidiary codes. The final list of codes was discussed within the research team, and organised in four overarching themes, each addressing a different aim of the study (figure 2, blue boxes).

\section{RESULTS}

For the purpose of this study, undergraduate surgical education was defined as any form of teaching opportunity targeted at facilitating the acquisition of essential skills and knowledge in the management of the surgical patient.

\section{Current state of training}

Many of the study participants agreed that the current landscape of basic surgical education is deficient at multiple levels. A recurring theme echoed in all but one interview included the apparent discrepancy between students' knowledge base and competence in basic surgical skills (BSS).

For example, as P2 stated, "Their knowledge is fantastic they really know their anatomy. On the other hand, their skills are not that superb, and the reason for that is that they haven't had enough practice during their medical school."

This point was further echoed by $\mathrm{P} 4$ who stated that "In the operating theatre I would say 20 per cent of the young surgeons don't know how to properly scrub up, their knowledge of aseptic technique can vary from zero to modest, and up to a third of them wouldn't know how to tie surgical knots."

On further questioning, most participants attributed this to a profound lack of opportunities; that is, even for students who actively seek to be taught and practise their BSS in clinical settings, they often struggle to succeed. Chiefly, access to opportunities was described as a 'postcode lottery'; that is, some students may be lucky enough to benefit from local teaching initiatives, while their 'unlucky' counterparts remain underexposed and arguably undertrained.

This issue was illustrated by $\mathrm{P} 6$ when he stated that "Differences in newly qualified doctors across medical schools raises the question of standardisation as well as whether the sheer numbers in some universities are making the harmonisation of curricula even harder."

Therefore, the first main conclusion concerned the lack of standardisation across medical schools which evidently creates an opportunity gap and unequal baseline competence levels. Despite these concerns, the consensus was that most doctors manage to catch-up with their skills acquisition during their postgraduate training.
Another dominant theme included the idea that medical students nowadays are victims of 'information overload'. Faced with an unprecedented number of learning resources, learning itself has become somewhat counterproductive. As elucidated by P6, what once used to be learnt adequately through a single textbook, now can be accessed through hundreds of textbooks and multiple learning platforms. This has made the 'learning' part of medical school inappropriately lengthier at the expense of the 'doing' part.

This point was raised by $\mathrm{P} 1$ who stated that "The increase in resources out of the hospital in the form of online libraries... is in a way discouraging students from actually spending time in the hospital where they should be."

P10 also re-iterated this point by stating that "There are so many amazing things online nowadays, however there is something to be said about being able to filter all this information, and that's where you need the teaching part."

An overlapping theme was that of the hospital as a place for learning. Six out of 10 participants raised an interesting point about the shifting mentalities of the public regarding students 'experimenting' on humans for the purpose of learning. It is no longer as acceptable to take chances on patients by letting students practise a skill, which is why simulation is taking up such an important role.

This argument was raised by P9 who stated that "The public is pressing towards a higher degree of supervision and subsequently the teachers are feeling increasingly uncomfortable to allow young trainees to practice on human bodies."

Moreover, part of the reason why the importance of the hospital is declining as a place for learning is the illdefined role of students within healthcare teams. Seven participants described students as 'mere observers' rather than active members of a team. There was overwhelming support over the idea that students should be assigned responsibilities and tasks with the appropriate supervision; lack of this, ultimately leads to disengagement and disinterest. In other words, students are deprived of traditional 'See one, do one' learning opportunities. While this does happen to some extent, this depends on the supervising consultant, which again highlights potential standardisation asymmetries across medical schools.

These asymmetries were noted by $\mathrm{P} 6$ who suggested, "There is this old-fashioned mentality which is so well embedded in people's minds that we should not be giving students actual duties and responsibilities. Of course, this cannot be done just by throwing them in there-but with professional supervision and guidance we can make them feel part of the team." 


\section{Challenges of introducing change}

Having identified gaps in current curricula, participants were invited to identify potential challenges one may face in an attempt to improve surgical education. Lack of time was the most dominant theme. Some participants raised an important point: if one was to introduce a more robust model of surgical education, this would surely compete with other topics of education both for time and space. Indeed, as much as surgeons want to refine their future colleagues before entering postgraduate training, so do psychiatrists and medics and in a 5-year period there is only a finite amount of time one could dedicate to individual topics.

This point was raised by P5 who stated, "I would obviously love to see more training in surgery but at the same time we have to appreciate the balance that the medical school has to offer, for all the different specialities. So even if I would like to see 4 years of medical school dedicated to surgery, I do realise that there is finite time to also teach neurology, psychiatry, radiology, etc."

Regarding the lack of time, interesting points were also raised about the very availability of clinical supervisors and how realistic it is to expect surgeons to spare the time to teach students in clinical environments.

An example of one of these points came from P9 who suggested that "We all work in pressurised environments in hospitals where people are working as efficiently as they can and often, they don't have the time to allow students to practice. Things have become so fast-paced that especially in busy tertiary centres, it has become impossible for students to learn in."

In view of the above findings, there was strong support over reorganising current curricula rather than simply introducing new, time-consuming and resource-draining courses. Views regarding the current curricula are best articulated by P10, suggesting that we shall work towards identifying and minimising moments of 'dead time', that is, periods in existing timetables not serving students' learning in any major way. In doing so, the improved curricula will then be more receptive to innovative models like IG4.

\section{Role of simulation}

Conversations regarding the apparent lack of opportunities for BSS, brought up the topic of SBL and the extent to which it can substitute learning in operating theatres. The consensus was that while simulation cannot replace BSS acquisition normally occurring in clinical settings, it can play a massive role in catalysing the transition of students from the 'learning' phase to the 'competency' phase of their learning curve.

The inability of SBL to substitute for BSS was noted by P4 who stated, "I've always felt that simulation training is very useful, but it doesn't compare to actually being in the OR dealing with real life cases. But it's a very good practice opportunity to start with. It makes you a little bit more confident when the real thing comes along."

A few participants also highlighted the potential of SBL to serve as a springboard for surgical specialities currently facing recruitment shortages; in that context, students are encouraged to learn more about the speciality and ultimately consider this as a career option. One informant suggested that by exposing students through simulation to aspects of care they are missing due to limited time in operating theatres, we can at least prepare them theoretically before they are first asked to apply themselves in postgraduate training.

This potential role for SBL was raised by $\mathrm{P} 8$ who suggested that "If the student sees the opportunity to have a week or two to in surgical skills simulation they may be encouraged to say 'I don't want to be a pure physician' or 'Yes, I want to be a surgeon'. In other words, we are also giving them some encouragement to think about their career development. That's number one for me."

Moreover, in view of the ever-rising competition in all aspects of training to become a surgeon, be it in residency applications through to training completion, the speed of skill acquisition along with the ability to adhere to short operative times are both becoming increasingly relevant. Overall, in this hypercompetitive environment most participants agreed that the concept of 'speed' is a crucial determinant of career progression both in terms of personal development and overall clinical performance.

\section{Appraisal of IG4}

To produce a comprehensive contextual analysis, we explored the participants' views of IG4 and its capacity to solve existing inefficiencies. As portrayed through earlier themes, existing teaching practices in medical schools are regarded as highly deficient.

Despite being at its infancy, IG4 was endorsed for its potential to solve what the professors described as a 'fundamental problem for aspiring surgeons'. Among the various issues that were explored, most participants commended its all-roundedness and holistic approach (it provided a good overall summary of key surgical themes), but also its inherent integration to clinical practice. Several interviewees commented that linking specific skills and knowledge to relevant clinical scenarios reinforces the learning benefits and the likelihood for more accurate recall in specific clinical circumstances.

The benefits of IG4 were raised by P5 when they stated that "The strengths of the model are obvious from its very structure: you can provide a holistic approach to surgery and produce the physician of the future the RCS has portrayed. If there's one way of achieving this, it is through at least testing it (the IG4)." 
This point was also raised by P1 who stated that "I think people are in danger of specialising too quickly and you're showing them all the different aspects of surgery and I thought it was excellent."

Most importantly, participants also discussed the versatility of the model and its capacity for international adaptation. Through 'informant triangulation ${ }^{15} 16$ we managed to validate the applicability of the model across different learning environments and geographical contexts; for example, UK, Greece and the USA. Participants particularly pointed out that being a standalone course, IG4 is transferable to many learning environments assuming the presence of basic simulation infrastructure. In other words, it may be provided to students either in the form of an elective or compulsory module in a similar way that other self-selected components are offered.

Lastly, regarding financial feasibility of projects like IG4, most participants agreed that for the implementation of such programmes across medical schools, there must be extensive planning and the expectation that in the short-run it will be costly. However, many supported that this would be offset as junior doctors will be better trained to take up advanced responsibilities in the long run.

For example, P2 suggested that "...like any other thing in education really is that we need to plant the seed and wait for it to grow. And that would take time even though it's costly."

\section{DISCUSSION}

While addressing the first focus area of this study, which was to explore the current state of undergraduate surgical training, it became evident that the current landscape of surgical education is suboptimal. Exploring these issues further, it became apparent that these are intrinsically linked to a global mentality shift, preventing students from benefiting under current education models. ${ }^{17} \mathrm{~A}$ key theme that arose was the increasing heterogeneity between, and in some cases within, medical schools in the design and implementation of the undergraduate surgical curriculum. In some instances, this has resulted in a situation where students are overburdened with different theoretical resources which do aid learning, but do not represent adequate substitutes for the 'doing' part of surgery. In other cases, opportunities for 'doing' are further constrained by changing attitudes around what is deemed acceptable for medical students to do while on rotations.

This lack of harmonisation, means that students are not afforded equal training opportunities, ultimately leading to discrepancies in preparedness for surgical posts as identified by Lee et al $2016 .{ }^{4}$ We concluded that important factors have progressively contributed towards the current state of affairs; the principle one being that medical schools are constrained for time. While it would be impractical to increase the time students spend in medical school, there is clearly a need to reshuffle existing curricula through the use of innovation in order to accommodate new learning needs in more time-efficient and cost-efficient methods. ${ }^{18}$

Interviews revealed another strong message regarding SBL: while it is undisputed that the best way to develop competency in BSS is by spending more time in operating theatres, SBL can play a critical role in facilitating the progression of students along the learning curve of BSS. Moreover, this raised interesting discussions about the apprenticeship model and whether it continues to form a viable model of teaching in the $21^{\text {st }}$ century. Our findings reinforce previously reported concerns about the 'demise of the firm', that is the gradual deterioration of traditional models of teaching which includes intergenerational learning, between novices and experienced healthcare professionals. ${ }^{19}$ These results are consistent with a breadth of research which has already established the importance of SBL to tackle this apparent change. ${ }^{82021}$

On top of this, we were able to elicit additional underappreciated and underreported benefits of SBL, such as its usefulness in exposing students to different surgical specialities and career opportunities, thereby helping to address longstanding recruitment problems. Given the aforementioned challenges, the main driver behind the Ci4R creation was the changing surgical landscape, requiring surgical trainees with the potential to adapt and evolve at times of mounting cost-pressures and reshuffled skill-sets. With more than 5 years of positive feedback, Ci4R has evolved into its latest version of IG4-a version this study appraised through expert opinion. Our participants agreed that the architecture employed in IG4 effectively tackles many of the above mentioned challenges. One can think of its multidimensional design by considering its three axes: $\mathrm{x}, \mathrm{y}, \mathrm{z}$. Modules situated on axes $\mathrm{x}$ and $\mathrm{y}$ aim to equip future surgeons with skills and knowledge, respectively, necessary for a sound surgical acumen. The third parameter in this new equation of IG4 is the $\mathrm{z}$ axis which in turn corresponds to a third metric - that of 'affinity'. Participants agreed that this new metric has transformed IG4 into a flexible framework, enabling educators to set up module 'clusters' with speciality-specific aims. This allows for the introduction of new module clusters without disrupting existing course components (eg, module clusters of existing surgical specialities).

In our appraisal of IG4, most participants agreed innovative teaching frameworks like ours can take the pressure off hospitals and relocate much of the teaching to friendlier environments like simulation labs where educators have the time and motivation to teach students in a holistic way. Importantly, IG4 successfully addresses many of the qualities highlighted in the most recent RCS report in the UK.

Lastly, as elucidated by the participants, IG4 allows for a more comprehensive immersion in previously underexplored specialities such that students can explore their 
full scope and appreciate their role in the management of surgical patients. Relevantly, according to Gelder et al, career choices are usually the product of longstanding interest, exposure to the job and working environment. ${ }^{22}$ The IG4 design, therefore, not only allows for isolated consideration of surgical specialities when it comes to choosing careers, but also helps students add a new unique dimension in their decision process: a structured and holistic 'feel' of the specialty-an element not reproducible via current medical school curricula.

\section{LIMITATIONS}

Use of qualitative evaluation of a curriculum alone is unlikely to form an adequate evidence base to inform educational policy. To mitigate this shortcoming, we deliberately used both participant and analyst triangulation to maximise the validity of our findings. Another limitation of this study may be the lack of exploration of student's perspectives on the curriculum; an area worth exploring in future work. However, incorporation of students' perspectives could have distorted the focus of our objectives and moved our project beyond the intended scope which was to gather expert opinion on the potential of the proposed curriculum to address existing gaps and inefficiencies in educational settings. It was felt that these gaps are more easily discernible to experienced educators as opposed to students who have only just embarked on their educational journey, and therefore were less likely to recognise such challenges. Future work could focus on capturing the views of educators in unexplored continents such as Asia and Australia, which could in turn provide new insights in the field.

\section{CONCLUSION}

The vision encapsulated by IG4 is to provide holistic surgical education structured in the form of a simple mathematical analogue. By coding and situating all current and future teaching modules on three-dimensional grid $(\mathrm{x}, \mathrm{y}, \mathrm{z})$, the aim is to produce a reproducible model of education which strikes two aims in a single shot. First, to relocate some of the learning that currently happens in hospitals into safer and more efficacious environments and second, to instil a sense of motivation and mentor students who are interested in surgery. Equally, IG4 can serve as a potent research platform for the appraisal of educational interventions; and this can include original studies as well as high quality evidence synthesis in arising research questions. Further to this, we believe that a systematic dissemination of IG4 across Europe can pave the way for the development of a bigger data set, which will be amenable to more sophisticated data analysis. This will provide accurate insight into inter-module interactions and therefore achieve further harmonisation of the curriculum map.

\section{Author affiliations}

${ }^{1}$ King's College London - Strand Campus, London, UK

${ }^{2}$ Women's Health Research Unit, Queen Mary University of London, London, UK
${ }^{3}$ Barts and The London School of Medicine and Dentistry, London, UK

${ }^{4}$ National and Kapodistrian University of Athens, Athinon, Greece

${ }^{5}$ Aristotle University of Thessaloniki, Thessaloniki, Greece

${ }^{6}$ Renal Transplant Directorate, Imperial College Healthcare NHS Trust, London, UK

${ }^{7}$ Department of Surgery and Cancer, Imperial College London, London, UK

${ }^{8}$ Harvard Medical School GHSM, Boston, Massachusetts, USA

${ }^{9}$ European University Cyprus, Nicosia, Nicosia, Cyprus

${ }^{10}$ Experimental Educational and Research Centre ELPEN, Athens, Greece

Contributors MS is responsible for the conception of the Integrated Generation 4 ESMSC curriculum. MS and AP are the primary leads of the ESMSC marathon course and share ownership of the course concept. IT designed the validation methods of the IG4 curriculum and drafted parts of the manuscript. MS co-designed and approved research methodology (validation). MS and IT are equal contributors for this manuscript. KL, MN, AD and EIE contributed in performing study and collecting the data. GT, VP and GCV are senior authors providing constructive feedback in the IG4 and the manuscript. All authors have approved final idea.

Funding The authors have not declared a specific grant for this research from any funding agency in the public, commercial or not-for-profit sectors.

Competing interests None declared.

Patient and public involvement statement This study did not include any patients. However, all the included subjects were qualified senior clinicians and academics, experts in the field of surgical education. The conclusions are addressed at the medical community, reflecting a future direction of medical education.

\section{Patient consent for publication Not required.}

Ethics approval The ESMSC course is compatible with the current 3R principles for animal-model simulation (refinement, replacement and reduction). Ethical approval was granted by the Hellenic Republic - Attica Region (Perifereia Attikis - PATT), Department of Animal Studies and Relevant Affairs and met directive $63 / 2010$, PD 56/April 2013. The license reference number is 4857/15-09-2017, MS, AP et al. All procedures performed in studies involving human participants were in accordance with the ethical standards of the institutional and/or national research committee and with the 1964 Helsinki declaration and its later amendments or comparable ethical standards. All applicable international, national and/or institutional guidelines for the care and use of animals were followed.

Provenance and peer review Not commissioned; externally peer reviewed.

Data availability statement Data are available upon reasonable request. Interview transcripts and thematic analysis can be made available upon reasonable request from the authors. Due to participant confidentiality, all data will be provided after removal of identifiable details.

Open access This is an open access article distributed in accordance with the Creative Commons Attribution Non Commercial (CC BY-NC 4.0) license, which permits others to distribute, remix, adapt, build upon this work non-commercially, and license their derivative works on different terms, provided the original work is properly cited, appropriate credit is given, any changes made indicated, and the use is non-commercial. See: http://creativecommons.org/licenses/by-nc/4.0/.

ORCID iD

Michail Sideris http://orcid.org/0000-0002-4199-6700

\section{REFERENCES}

1 Jayakumar N, Brunckhorst O, Dasgupta P, et al. E-Learning in surgical education: a systematic review. J Surg Educ 2015;72:1145-57.

2 Sideris M, Papalois A, Theodoraki K, et al. Promoting undergraduate surgical education: current evidence and students' views on ESMSC international wet lab course. J Invest Surg 2017;30:71-7.

3 Schmidt LE, Cooper CA, Guo WA. Factors influencing US medical students' decision to pursue surgery. J Surg Res 2016;203:64-74.

4 Lee MJ, Drake TM, Malik TAM, et al. Has the bachelor of surgery left medical school? - a national undergraduate assessment. J Surg Educ 2016;73:655-9.

5 Pitruzzella B, Leahy P. Future of surgery, 2018.

6 Scally CP, Minter RM. Medical school training for the surgeon. Surg Clin North Am 2016;96:1-13.

7 Khoushhal Z, Hussain MA, Greco E, et al. Prevalence and causes of attrition among surgical residents: a systematic review and metaanalysis. JAMA Surg 2017;152:265-72. 
8 Theodoulou I, Nicolaides M, Athanasiou T, et al. Simulation-based learning strategies to teach undergraduate students basic surgical skills: a systematic review. J Surg Educ 2018;75:1374-88.

9 Sideris M, Papalois A, Tsoulfas G, et al. Developing an international combined applied surgical science and wet lab simulation course as an undergraduate teaching model. Biomed Res Int 2015;2015:1-10.

10 Sideris M, Hanrahan J, Tsoulfas G, et al. Developing a novel international undergraduate surgical masterclass during a financial crisis: our 4-year experience. Postgrad Med J 2018;94:263-9.

11 Hasson F, Keeney S, McKenna H. Research guidelines for the Delphi survey technique. J Adv Nurs 2000;32:1008-15.

12 McKenna HP. The Delphi technique: a worthwhile research approach for nursing? J Adv Nurs 1994;19:1221-5.

13 Palinkas LA, Horwitz SM, Green CA, et al. Purposeful sampling for qualitative data collection and analysis in mixed method implementation research. Adm Policy Ment Health 2015;42:533-44.

14 Castleberry A, Nolen A. Thematic analysis of qualitative research data: is it as easy as it sounds? Curr Pharm Teach Learn 2018;10:807-15.
15 Denzin NK. Triangulation: a case for methodological evaluation and combination. Sociological methods 1978:339-57.

16 Patton MQ. Enhancing the quality and credibility of qualitative analysis. Health services research 1999;34:1189.

17 Neylan CJ, Nelson EF, Dumon KR, et al. Medical school surgical boot camps: a systematic review. J Surg Educ 2017;74:384-9.

18 Asch DA, Weinstein DF. Innovation in medical education. New England Journal of Medicine 2014;371:794-5.

19 Timm A. The demise of the firm-What is happening to apprenticeship learning, 2013.

20 McGaghie WC, Issenberg SB, Petrusa ER, et al. Effect of practice on standardised learning outcomes in simulation-based medical education. Med Educ 2006;40:792-7.

21 Konge L, Lonn L. Simulation-Based training of surgical skills. Perspect Med Educ 2016;5:3-4

22 Gelder C, Selwyn-Gotha J, Rouhani Ml, et al. Which factors influence career choice? A medical student survey. Int J Surg 2016;36:S103-4. 